CONFORMAL GEOMETRY AND DYNAMICS

An Electronic Journal of the American Mathematical Society

Volume 2, Pages 25-28 (February 11, 1998)

S $1088-4173(98) 00025-3$

\title{
VOLUME FORMULAE FOR REGULAR HYPERBOLIC CUBES
}

\author{
T. H. MARSHALL
}

\begin{abstract}
We express the volume of a regular cube in hyperbolic $n$-space as an integral on $[0, \infty)$, and derive from this an asymptotic volume formula for the regular ideal hyperbolic $n$-cube. This in turn is applied to finding an asymptotic lower bound for the least number of simplices into which a Euclidean $n$-cube can be triangulated.
\end{abstract}

\section{INTRODUCTION}

Three types of regular solid, namely the simplex, cube and crosspolytope (generalized octahedron), occur in spaces of all dimensions. In particular each of these solids is realized in hyperbolic $n$-space as a regular ideal polyhedron, which we denote by $\overline{T_{n}}, \overline{C_{n}}$ and $\overline{G_{n}}$ respectively. For the first and last of these we have the asymptotic volume formulae

$$
\begin{gathered}
\operatorname{Volume}\left(\overline{T_{n}}\right) \approx e \sqrt{n} / n !, \\
\operatorname{Volume}\left(\overline{G_{n}}\right) \approx e 2^{n} / n !
\end{gathered}
$$

(where $\mathrm{f}(n) \approx \mathrm{g}(n)$ means that $\mathrm{f}(n) / \mathrm{g}(n) \rightarrow 1$ as $n \rightarrow \infty)$. The first of these is originally due to Milnor [3] (see also [2] and [5]), and the second to W. D. Smith [5]. Smith also gives a lower bound for Volume $\left(\overline{C_{n}}\right)$ and conjectures the existence of a positive constant $L$, for which

$$
\lim _{n \rightarrow \infty} n^{1 / 2} \operatorname{Volume}\left(\overline{C_{n}}\right)^{1 / n}=L .
$$

In this note we give an asymptotic volume formula for $\overline{C_{n}}$, similar to (1) and (2), from which (3) and an explicit evaluation of $L$ follow. In fact we find an exact expression for the volume of a regular cube of any edge length, as an integral on $[0, \infty)$, and then derive the asymptotic using Laplace's method.

One reason that the volume of $\overline{C_{n}}$ is of interest is that it gives information about the "simplexity" of a Euclidean $n$-cube, that is, the smallest number of simplices into which it can be triangulated. We denote this number by $\phi(n)$. Trivially, $\phi(1)=1$ and $\phi(2)=2$, and it is known that $\phi(3)=5$ and $\phi(4)=16$. For $n \geq 5$ the values of $\phi(n)$ are unknown.

Hyperbolic geometry can be used to find a lower bound for $\phi(n)$ in the following way, described in [5]. If we inscribe the Euclidean $n$-cube in a sphere, it represents a regular ideal hyperbolic cube in the Klein (projective) model, and the simplices

Received by the editors August 15, 1997 and, in revised form, November 26, 1997.

1991 Mathematics Subject Classification. Primary 51M10, 51M25, 52A35, 52A38; Secondary 05B45, 51M20, 52A40.

Key words and phrases. Hyperbolic cube, volume, simplex, triangulation.

(C)1998 American Mathematical Society 
of the decomposition represent hyperbolic simplices. It follows that $\phi(n)$ must be at least the hyperbolic volume of $\overline{C_{n}}$ divided by that of the of largest simplex contained in $\overline{C_{n}}$. By the theorem of Haagerup and Munkholm [2], the maximum possible volume of a hyperbolic $n$-simplex is attained by a regular ideal $n$-simplex $\Delta_{n}$, and so, by $(1), \phi(n)$ has asymptotic lower bound $n$ ! Volume $\left(\overline{C_{n}}\right) /\left(e n^{1 / 2}\right)$ (i.e., $\underline{\lim \phi}(n)\left(e n^{1 / 2}\right) /\left(n\right.$ !Volume $\left.\left.\left(\overline{C_{n}}\right)\right) \geq 1\right)$. Thus our asymptotic for $\overline{C_{n}}$ immediately gives an asymptotic lower bound for $\phi(n)$. Further discussion of bounds for $\phi(n)$ can be found in [5].

The results in this paper have been proved independently by W. D. Smith.

\section{ThEOREMS}

Let $C_{n}(\lambda)$ be the regular hyperbolic $n$-cube, represented in the Klein model by a cube centred at the origin with Euclidean edge length $2 \lambda / \sqrt{n}$. The parameter $\lambda$ thus lies in $(0,1]$ and $\lambda=1$ gives the ideal regular cube. In hyperbolic terms, $\lambda=(\tanh d) \sqrt{n}$, where $d$ is the hyperbolic distance from the centre of $C_{n}(\lambda)$ to the centre of any of its faces (see e.g. [4], chapter 6).

We prove

\section{Theorem 1.}

$$
\operatorname{Volume}\left(C_{n}(\lambda)\right)=\frac{2^{n+1} \sqrt{n}}{\lambda \Gamma\left(\frac{n+1}{2}\right)} \int_{0}^{\infty}\left[e^{-u^{2} / \lambda^{2}} h(u)\right]^{n} \mathrm{~d} u
$$

where

$$
h(u)=\int_{0}^{u} e^{x^{2}} \mathrm{~d} x
$$

\section{Corollary 2.}

$$
\operatorname{Volume}\left(\overline{C_{n}}\right) \approx \sqrt{2} L^{n} n^{-n / 2} \quad(n \rightarrow \infty)
$$

where $L=(2 e)^{1 / 2} / k=2.52304 \ldots$ and $k$ is the (unique) critical point of $e^{-u^{2}} h(u)$.

We have thus identified the constant in (3).

In view of the discussion in the introduction, (1), (6) and Stirling's formula give

Corollary 3. Asymptotically $\phi(n)$ is at least

$$
(2 \sqrt{\pi} / e)(L / e)^{n} n^{n / 2} \quad(n \rightarrow \infty) .
$$

Theorem 1 is a particular case of the following result about the integral over a cube of the radially symmetric function $F\left(r^{2}\right)$ defined by

$$
F(s)=\int_{0}^{\infty} f(t) e^{s t} \mathrm{~d} t
$$

so that $F$ is essentially a Laplace transform.

Theorem 4. If $F(s)$ is as above, and the function $(t, \mathbf{x}) \rightarrow f(t) e^{|\mathbf{x}|^{2} t}$ is integrable on $[0, \infty) \times[0, l]^{n}$, then

$$
\int_{[0, l]^{n}} F\left(|\mathbf{x}|^{2}\right) \mathrm{d} x_{1} \ldots \mathrm{d} x_{n}=2 l^{n-2} \int_{0}^{\infty} u f\left(u^{2} / l^{2}\right)(h(u) / u)^{n} \mathrm{~d} u .
$$


Proof. By Fubini's theorem we have

$$
\begin{aligned}
\int_{[0, l]^{n}} F\left(|\mathbf{x}|^{2}\right) & =\int_{[0, l]^{n}}\left[\int_{0}^{\infty} f(t) e^{|\mathbf{x}|^{2} t} \mathrm{~d} t\right] \mathrm{d} x_{1} \mathrm{~d} x_{2} \ldots \mathrm{d} x_{n} \\
& =\int_{0}^{\infty} f(t)\left[\int_{[0, l]^{n}} e^{|\mathbf{x}|^{2} t} \mathrm{~d} x_{1} \mathrm{~d} x_{2} \ldots \mathrm{d} x_{n}\right] \mathrm{d} t \\
& =\int_{0}^{\infty} f(t)\left[\int_{0}^{l} e^{x^{2} t} \mathrm{~d} x\right]^{n} \mathrm{~d} t \\
& =\int_{0}^{\infty} f(t)\left[t^{-1 / 2} h\left(t^{1 / 2} l\right)\right]^{n} \mathrm{~d} t .
\end{aligned}
$$

The substitution $u=t^{1 / 2} l$ then gives (8).

Remark. By the same argument we obtain a modified version of (8), where the signs of the exponents in the integrands of both (5) and (7) are changed, that is, when $F(s)$ is the Laplace transform of $f(t)$.

Proof of Theorem 1. Since the volume element in the Klein model is

$$
\left(1-|\mathbf{x}|^{2}\right)^{-(n+1) / 2} \mathrm{~d} x_{1} \mathrm{~d} x_{2} \ldots \mathrm{d} x_{n},
$$

and using the symmetry of $C_{n}$, we have

$$
\operatorname{Volume}\left(C_{n}(\lambda)\right)=2^{n} \int_{[0, \lambda / \sqrt{n}]^{n}}\left(1-|\mathbf{x}|^{2}\right)^{-(n+1) / 2} \mathrm{~d} x_{1}, \ldots \mathrm{d} x_{n} .
$$

Since

$$
(1-s)^{-(n+1) / 2}=\int_{0}^{\infty} \frac{t^{(n-1) / 2} e^{-t}}{\Gamma\left(\frac{n+1}{2}\right)} e^{s t} \mathrm{~d} t \quad(s<1),
$$

Theorem 1 follows from Theorem 4 .

Proof of Corollary 2. Let $\phi(u)=e^{-u^{2}} h(u)$ and $j(u)=\log (\phi(u))$. Let $\xi$ be any stationary point of $\phi(u)$ in $[0, \infty)$. We have

$$
\phi^{\prime}(u)=1-2 u \phi(u) \quad \text { and } \quad \phi^{\prime \prime}(u)=-2\left(u \phi^{\prime}(u)+\phi(u)\right),
$$

whence $\xi$ is a solution of $2 u \phi(u)=1, \phi(\xi)=1 / 2 \xi, \phi^{\prime \prime}(\xi)=-2 \phi(\xi)$, and $j^{\prime \prime}(\xi)=$ $\phi^{\prime \prime}(\xi) / \phi(\xi)=-2$. Hence all stationary points of $\phi(u)$ are maxima. Since, in addition, $\phi(0)=0$ and $\phi(u) \rightarrow 0$ as $u \rightarrow \infty$, there is a unique stationary point $k$ and $\phi(u)$ attains its maximum on $[0, \infty)$ at $k$.

We now set $\lambda=1$. The integrand in (4) is $\exp (n j(u))$ and so, by Laplace's method (see e.g. [1], §4.2]), the integral is asymptotically

$$
\phi(k)^{n}(2 \pi)^{1 / 2}\left(-n j^{\prime \prime}(k)\right)^{-1 / 2} \approx(2 k)^{-n}(\pi / n)^{1 / 2} \quad(n \rightarrow \infty) .
$$

In conjunction with Stirling's formula, this gives (6).

Remark. One can obtain similar asymptotics for the volume of $C_{n}(\lambda)$ as $n \rightarrow \infty$ where $\lambda$ takes any fixed value in $(0,1]$. 


\section{REFERENCES}

1. N.G. De Bruijn, Asymptotic Methods in Analysis, North-Holland, 1970.

2. U. Haagerup and H. J. Munkholm, Simplices of Maximal Volume in Hyperbolic n-Space, Acta Math. 147 (1981), 1-11. MR 82j:53116

3. J. W. Milnor, 'How to Compute Volume in Hyperbolic Space' in Collected Papers Vol. 1, Geometry (Publish or Perish, 1994).

4. J. G. Ratcliffe, Foundations of Hyperbolic Manifolds, Springer-Verlag, 1994. MR 95j:57011

5. W. D. Smith, Studies in Computational Geometry Motivated by Mesh Generation, Thesis, Princeton, 1989.

Department of Mathematics, University of Auckland, Private Bag 92019, Auckland, New Zealand

E-mail address: t_marshall@math.auckland.ac.nz 